\title{
Anomalous Effects of "Guest" Charges Immersed in Electrolyte: Exact 2D Results
}

\author{
L. Šamaj ${ }^{1}$
}

October 30, 2018

\begin{abstract}
We study physical situations when one or two "guest" arbitrarilycharged particles are immersed in the bulk of a classical electrolyte modelled by a Coulomb gas of \pm unit point-like charges, the whole system being in thermal equilibrium. The models are treated as twodimensional with logarithmic pairwise interactions among charged constituents; the (dimensionless) inverse temperature $\beta$ is considered to be smaller than 2 in order to ensure the stability of the electrolyte against the collapse of positive-negative pairs of charges. Based on recent progress in the integrable $(1+1)$-dimensional sine-Gordon theory, exact formulas are derived for the chemical potential of one guest charge and for the asymptotic large-distance behavior of the effective interaction between two guest charges. The exact results imply, under certain circumstances, anomalous effects such as an effective attraction (repulsion) between like-charged (oppositely-charged) guest particles and the charge inversion in the electrolyte vicinity of a highlycharged guest particle. The adequacy of the concept of renormalized charge is confirmed in the whole stability region of inverse temperatures and the related saturation phenomenon is revised.
\end{abstract}

KEY WORDS: Coulomb systems; logarithmic interactions; charge inversion; renormalized charge; sine-Gordon model.

${ }^{1}$ Institute of Physics, Slovak Academy of Sciences, Dúbravská cesta 9, 84511 Bratislava, Slovak Republic; e-mail: fyzimaes@savba.sk 


\section{Introduction}

This paper deals with physical situations when one or two "guest" charges, say arbitrarily charged colloidal particles with a hard core of radius $\sigma$, are immersed in a classical electrolyte modelled by an infinite Coulomb gas of positive/negative unit charges. In order to obtain explicit results we consider the point-like limit of the guest charges, i.e. $\sigma / \lambda \rightarrow 0$ where $\lambda$ is a characteristic correlation length of electrolyte species; the obtained results are not expected to be applicable to large-sized colloids. If the charges of the guest particles are sufficiently large, anomalous counterintuitive phenomena emerge in the system [1].

One of such phenomena is the appearance, under some circumstances, of an effective (i.e., mediated by the electrolyte) attraction between likecharged colloids. While the traditional DLVO theory [2, 3] always predicts an effective repulsion between two like-charged colloids [4, 5], experimental measurements [6, 7] and numerical simulations [8, 9] provide evidence for attraction, especially within confined geometries (close to a dielectric wall or between two glass plates) but also in the bulk of the electrolyte [10]. It was argued that the effective attraction of two like-charged colloids in the presence of a single wall can arise also from a non-equilibrium hydrodynamic effect [11, 12].

Another interesting effect is the overcharging, or the charge inversion, of a highly charged colloid [13. This effect occurs when the number of electrolyte counterions in the vicinity of the colloidal surface becomes so high that the colloidal bare charge is locally overcompensated. The charge inversion has been observed experimentally by electrophoresis [14] and in simulations [15]. Its theoretical explanation is based on Wigner-crystal theories [16, 17.

The latter effect is related to the concept of renormalized charge [18, 19, 20, 21, 22, 23. The true electric potential far from the colloid immersed in a weakly-coupled electrolyte is supposed to exhibit the Debye-Hückel form, but with a renormalized-charge prefactor which is different from the bare charge of the colloid. An important feature, which occurs in the framework of the nonlinear Poisson-Boltzmann equation, is that the renormalized charge saturates monotonically at some finite value when the colloidal bare charge goes to infinity [22, 23]. Monte-Carlo simulations of a salt-free colloidal cell model 24] indicate the existence of a maximum in the plot of the renormalized charge versus the bare colloidal charge. Téllez and Trizac [25] considered the possibility of a more general phenomenon of potential saturation.

A theoretical elucidation of anomalous phenomena requires to go beyond mean-field approximations by incorporating electrostatic correlations among electrolyte particles. Heuristic phenomenological approaches applied so far are based on plausible, but not rigorously justified, arguments. Some exactly solvable models are needed. The best candidates are two-dimensional (2D) 
Coulomb systems with logarithmic pairwise interactions among the charged constituents. The 2D Coulomb gas of \pm unit point-like charges is stable against the collapse of positive-negative pairs of charges at high enough temperatures, namely for $\beta<2$ where $\beta$ is the (dimensionless) inverse temperature or coupling constant. The collapse point $\beta=2$, at which the collapse starts to occur, is equivalent to the free-fermion point of the Thirring representation of the 2D Coulomb gas [26, 27]; although the free energy and the particle density diverge, the truncated Ursell correlation functions are finite at this point. In a recent work [28, we have solved exactly the 2D problem of one colloid immersed in the Coulomb gas just at the free-fermion point. An explicit form of the induced electric potential as a function of the bare colloidal charge was derived at every point of the space. Based on this exact result, the concept of renormalized charge was shown to fail in this strong-coupling regime. On the other hand, the anticipated phenomenon of the electric potential saturation was confirmed at the free-fermion point.

Our present aim is to extend the exact treatment of the guest-charge(s) problem to the whole Coulomb-gas stability region of inverse temperatures $0 \leq \beta<2$. A first important step towards this aim has already been done by solving exactly the equilibrium statistical mechanics of the 2D Coulomb gas in the stability regime (the bulk thermodynamics, special cases of the surface thermodynamics and the large-distance behavior of the two-body correlation functions) via an equivalence with the integrable 2D Euclidean sine-Gordon theory; for a short review, see ref. [29]. As is shown in this paper, the problem of one (two) guest charge(s) immersed in the Coulomb plasma is related to the evaluation of one-point (two-point) expectation values of the exponential field in the sine-Gordon theory. Based on recent progress in the latter topic, we derive explicit formulas for the chemical potential of one guest charge and for the asymptotic large-distance behavior of the effective interaction between two guest charges. The exact results imply, under some circumstances, an effective attraction (repulsion) between like-charged (oppositely-charged) guest particles and the charge inversion in the electrolyte around a highly-charged guest particle. The adequacy of the concept of renormalized charge is confirmed in the whole stability region $0 \leq \beta<2$. The related saturation phenomenon is revised.

The paper is organized as follows. Basic facts about the bulk properties of the 2D symmetric Coulomb gas are summarized in Section 2. Section 3 deals with the problem of one guest charge in the electrolyte. The effective interaction between two guest charges immersed in the electrolyte is studied within a form-factor method for the equivalent sine-Gordon model in Section 4. Based on the exact results of Section 4, the concept of renormalized charge and the related saturation phenomenon are tested in Section 5. A brief recapitulation and some concluding remarks are given in Section 6 . 


\section{Basic facts about the 2D Coulomb gas}

We consider an infinite $2 \mathrm{D}$ plane $\Lambda$ of points $\mathbf{r} \in R^{2}$, filled with a homogeneous medium of dielectric constant $=1$. The electrostatic potential $v$ at a point $\mathbf{r}$, induced by a unit charge at the origin $\mathbf{0}$, is given by the 2D Poisson equation

$$
\Delta v(\mathbf{r})=-2 \pi \delta(\mathbf{r})
$$

The solution of this equation, subject to the boundary condition $\nabla v(\mathbf{r}) \rightarrow 0$ as $|\mathbf{r}| \rightarrow \infty$, reads

$$
v(\mathbf{r})=-\ln \left(\frac{|\mathbf{r}|}{r_{0}}\right), \quad \mathbf{r} \in R^{2}
$$

The free length constant $r_{0}$ will be set for simplicity to unity. This definition of the Coulomb potential in 2D maintains many generic properties (e.g., sum rules [30]) of "real" 3D Coulomb fluids with the interaction potential $v(\mathbf{r})=1 /|\mathbf{r}|, \mathbf{r} \in R^{3}$.

The symmetric Coulomb gas consists of two species of point-like particles with opposite unit charges $q_{j} \in\{+1,-1\}$; to simplify the notation, the elementary charge $e$ is set to unity. The bulk properties of the system in thermodynamic equilibrium are usually treated within the grand canonical ensemble. The ensemble is characterized by the (dimensionless) inverse temperature $\beta$, which plays the role of the coupling constant, and by the couple of equivalent particle fugacities $z_{+}=z_{-}=z$. Since the length scale $r_{0}$ in (2.2) was set to unity, the true dimension of $z$ is $[\text { length }]^{-2+(\beta / 2)}$. The grand partition function of the plasma is defined by

$$
\Xi(z)=\sum_{N_{+}, N_{-}=0}^{\infty} \frac{1}{N_{+} ! N_{-} !} \int_{\Lambda} \prod_{j=1}^{N}\left[\mathrm{~d}^{2} r_{j} z_{q_{j}}\right] \exp \left\{-\beta \sum_{j<k} q_{j} q_{k} v\left(\left|\mathbf{r}_{j}-\mathbf{r}_{k}\right|\right)\right\}
$$

where $N_{+}\left(N_{-}\right)$is the number of positively (negatively) charged particles and $N=N_{+}+N_{-}$. The system is stable against the collapse of positivenegative pairs of unit point-like charges provided that the corresponding Boltzmann factor $r^{-\beta}$ is integrable at short distances in $2 \mathrm{D}$, i.e. for $\beta<2$. In what follows, we shall restrict ourselves to this stability region of coupling constants.

To introduce the averaged many-particle densities, we denote by $\langle\cdots\rangle_{\beta}$ the standard thermal average. At the one-particle level, one considers the number density of particles of one sign

$$
n_{q}(\mathbf{r})=\left\langle\sum_{j} \delta_{q, q_{j}} \delta\left(\mathbf{r}-\mathbf{r}_{j}\right)\right\rangle_{\beta}, \quad q= \pm 1
$$

Due to the charge symmetry and space homogeneity, $n_{+}=n_{-}=n / 2$ where $n$ is the total density of particles. At the two-particle level, one considers 
the two-body number densities

$$
n_{q q^{\prime}}\left(\mathbf{r}, \mathbf{r}^{\prime}\right)=\left\langle\sum_{j \neq k} \delta_{q, q_{j}} \delta\left(\mathbf{r}-\mathbf{r}_{j}\right) \delta_{q^{\prime}, q_{k}} \delta\left(\mathbf{r}^{\prime}-\mathbf{r}_{k}\right)\right\rangle_{\beta}, \quad q, q^{\prime}= \pm 1
$$

which are translationally invariant, $n_{q q^{\prime}}\left(\mathbf{r}, \mathbf{r}^{\prime}\right) \equiv n_{q q^{\prime}}\left(\left|\mathbf{r}-\mathbf{r}^{\prime}\right|\right)$. The two-body densities decouple at asymptotically large distance onto the product of the corresponding one-body densities, $\lim _{r \rightarrow \infty} n_{q q^{\prime}}(r)=n_{q} n_{q^{\prime}}$. It is therefore natural to introduce the Ursell functions, $U_{q q^{\prime}}(r)=n_{q q^{\prime}}(r)-n_{q} n_{q^{\prime}}$, which go to 0 as $r \rightarrow \infty$. It is also useful to consider the pair distribution functions $g_{q q^{\prime}}(r)=n_{q q^{\prime}}(r) /\left(n_{q} n_{q^{\prime}}\right)$.

The short-distance behavior of the two-body densities is dominated by the Boltzmann factor of the corresponding pair Coulomb potential [31, 32]. In particular, the pair distribution functions behave like

$$
\begin{aligned}
g_{q q^{\prime}}(r) & \sim C_{q q^{\prime}} r^{\beta q q^{\prime}} \quad \text { as } r \rightarrow 0 \\
C_{q q^{\prime}} & =\exp \left[\beta\left(\mu_{q}^{\mathrm{ex}}+\mu_{q^{\prime}}^{\mathrm{ex}}-\mu_{q+q^{\prime}}^{\mathrm{ex}}\right]\right.
\end{aligned}
$$

Here, the excess (i.e. over ideal) chemical potential of the Coulomb-gas species is defined by

$$
\exp \left(\beta \mu_{q}^{\mathrm{ex}}\right)=\frac{z_{q}}{n_{q}}, \quad q= \pm 1
$$

and $\mu_{Q}^{\mathrm{ex}}$ with arbitrarily-valued $Q$ represents an extended definition of the excess chemical potential: $\mu_{Q}^{\mathrm{ex}}$ is the reversible work which has to be done in order to bring a particle of charge $Q$ (in units of the elementary charge e) from infinity into the bulk interior of the considered Coulomb gas. In the case of oppositely-charged particles, formula (2.6) reduces to

$$
g_{+-}(r) \sim\left(\frac{z_{+}}{n_{+}}\right)\left(\frac{z_{-}}{n_{-}}\right) r^{-\beta} \quad \text { as } r \rightarrow 0
$$

According to Eq. (2.1),$-\Delta /(2 \pi)$ is the inverse operator of the Coulomb potential $v(\mathbf{r})$. The grand partition function of the 2D Coulomb gas (2.3) can be thus turned via the Hubbard-Stratonovich transformation (see e.g. ref. [33]) into

$$
\Xi=\frac{\int \mathcal{D} \phi \exp [-S(z)]}{\int \mathcal{D} \phi \exp [-S(0)]}
$$

with

$$
S(z)=-\int_{\Lambda} \mathrm{d}^{2} r\left[\frac{1}{16 \pi} \phi \Delta \phi+2 z \cos (b \phi)\right], \quad b^{2}=\frac{\beta}{4}
$$

being the 2D Euclidean action of the sine-Gordon model. Here, $\phi(\mathbf{r})$ is a real scalar field and $\int \mathcal{D} \phi$ denotes the functional integration over this field. The 
fugacity $z$ is renormalized by the (diverging) self-energy term $\exp [\beta v(0) / 2]$, without changing the $z$-notation. The one- and two-body densities of the charged particles in the plasma are expressible as averages over the sineGordon action as follows

$$
n_{q}=z_{q}\left\langle\mathrm{e}^{\mathrm{i} q b \phi}\right\rangle, \quad n_{q q^{\prime}}\left(\left|\mathbf{r}-\mathbf{r}^{\prime}\right|\right)=z_{q} z_{q^{\prime}}\left\langle\mathrm{e}^{\mathrm{i} q b \phi(\mathbf{r})} \mathrm{e}^{\mathrm{i} q^{\prime} b \phi\left(\mathbf{r}^{\prime}\right)}\right\rangle
$$

With regard to Eq. (2.8), it holds

$$
\exp \left(-\beta \mu_{q}^{\mathrm{ex}}\right)=\left\langle\mathrm{e}^{\mathrm{i} q b \phi}\right\rangle, \quad q= \pm 1
$$

The short-distance behavior (2.9) is equivalent to

$$
\left\langle\mathrm{e}^{\mathrm{i} b \phi(\mathbf{r})} \mathrm{e}^{-\mathrm{i} b \phi\left(\mathbf{r}^{\prime}\right)}\right\rangle \sim\left|\mathbf{r}-\mathbf{r}^{\prime}\right|^{-4 b^{2}} \quad \text { as }\left|\mathbf{r}-\mathbf{r}^{\prime}\right| \rightarrow 0
$$

Under this conformal normalization of the exponential field, the divergent self-energy factor (which renormalizes $z$ ) disappears from statistical relations calculated within the sine-Gordon representation. The short-distance formula (2.14) is the special case of a more general relation

$$
\left\langle\mathrm{e}^{\mathrm{i} a \phi(\mathbf{r})} \mathrm{e}^{\mathrm{i} a^{\prime} \phi\left(\mathbf{r}^{\prime}\right)}\right\rangle \sim\left\langle\mathrm{e}^{\mathrm{i}\left(a+a^{\prime}\right) \phi}\right\rangle\left|\mathbf{r}-\mathbf{r}^{\prime}\right|^{4 a a^{\prime}} \quad \text { as }\left|\mathbf{r}-\mathbf{r}^{\prime}\right| \rightarrow 0
$$

valid in a restricted region of the parameters $a$ and $a^{\prime}$ such that the one-point average $\left\langle\mathrm{e}^{\mathrm{i}\left(a+a^{\prime}\right) \phi}\right\rangle$ be finite.

The sine-Gordon model (2.11) is integrable 34]. Its particle spectrum consists of one soliton-antisoliton pair $(S, \bar{S})$ with equal masses $M$ and of $S-\bar{S}$ bound states ("breathers") $\left\{B_{j} ; j=1,2, \ldots<1 / \xi\right\}$ whose number at a given $b^{2}$ depends on the inverse of the parameter

$$
\xi=\frac{b^{2}}{1-b^{2}} \quad\left(=\frac{\beta}{4-\beta}\right)
$$

The mass of the $B_{j}$-breather is given by

$$
m_{j}=2 M \sin \left(\frac{\pi \xi}{2} j\right)
$$

and this breather disappears from the spectrum just when $m_{j}=2 M$. The breathers exist only in a subinterval of the stability region $0 \leq b^{2}<1 / 2$ $(0 \leq \beta<2)$ of the point-like Coulomb gas. The lightest $B_{1}$-breather, usually called the elementary one, has the mass

$$
m_{1}=2 M \sin \left(\frac{\pi \xi}{2}\right)
$$

and disappears from the particle spectrum just at the free-fermion point $b^{2}=1 / 2(\beta=2)$. The soliton-antisoliton pair is present in the spectrum 
up to the Kosterlitz-Thouless transition point $b^{2}=1(\beta=4)$ at which the sine-Gordon theory ceases to be massive.

The (dimensionless) specific grand potential $\omega$ of the 2D Euclidean sineGordon model, defined by

$$
-\omega=\lim _{|\Lambda| \rightarrow \infty} \frac{1}{|\Lambda|} \ln \Xi
$$

was found in ref. 35 by using the Thermodynamic Bethe ansatz:

$$
-\omega=\frac{m_{1}^{2}}{8 \sin (\pi \xi)}
$$

Under the conformal normalization of the exponential fields (2.14), the relationship between the fugacity $z$ and the soliton/antisoliton mass $M$ was established in ref. 36],

$$
z=\frac{\Gamma\left(b^{2}\right)}{\pi \Gamma\left(1-b^{2}\right)}\left[M \frac{\sqrt{\pi} \Gamma((1+\xi) / 2)}{2 \Gamma(\xi / 2)}\right]^{2-2 b^{2}}
$$

where $\Gamma$ stands for the Gamma function. Note that the mass $M$ has dimension of an inverse length. As a consequence of Eqs. (2.20) and (2.21), one has

$$
\left\langle\mathrm{e}^{\mathrm{i} b \phi}\right\rangle=\frac{1}{2} \frac{\partial(-\omega)}{\partial z}=\frac{M^{2}}{8 z\left(1-b^{2}\right)} \operatorname{tg}\left(\frac{\pi \xi}{2}\right)
$$

Relations (2.21) and (2.22), together with the equality

$$
\frac{n}{2 z}=\left\langle\mathrm{e}^{\mathrm{i} b \phi}\right\rangle
$$

determine explicitly the density-fugacity relationship and consequently the complete thermodynamics of the 2D Coulomb gas in the stability region [37.

\section{One guest charge in the electrolyte}

Let us consider a point-like particle of charge $Q$ with $Q$ being an arbitrarily valued real number; when $Q$ is interpreted as the valence it has to be an integer. The charge is put into the bulk interior of the $2 \mathrm{D}$ electrolyte, say at the origin $\mathbf{0}$. The electrostatic potential induced by the guest charge at a point $\mathbf{r} \in R^{2}$ is equal to $-Q \ln |\mathbf{r}|$. Its effect on the constant species fugacities $z_{ \pm}$is the following: $z_{q} \rightarrow z_{q}^{(1)}(\mathbf{r})=z|\mathbf{r}|^{\beta Q q}$. The excess chemical potential of the guest particle is given by

$$
\exp \left(-\beta \mu_{Q}^{\mathrm{ex}}\right)=\frac{\Xi\left[z_{q}^{(1)}(\mathbf{r})\right]}{\Xi(z)}
$$


where $\Xi\left[z_{q}^{(1)}(\mathbf{r})\right]$ represents an obvious functional generalization of the definition (2.3) of the grand partition function with position-dependent particle fugacities. Performing the Hubbard-Stratonovich transformation, the sineGordon representation of $\Xi\left[z_{q}^{(1)}(\mathbf{r})\right]$ takes the standard form of Eq. (2.10) with the action

$$
S^{(1)}(z)=-\int_{\Lambda} \mathrm{d}^{2} r\left(\frac{1}{16 \pi} \phi \Delta \phi+z|\mathbf{r}|^{4 Q b^{2}} \mathrm{e}^{\mathrm{i} b \phi}+z|\mathbf{r}|^{-4 Q b^{2}} \mathrm{e}^{-\mathrm{i} b \phi}\right)
$$

We first shift the scalar field $\phi(\mathbf{r}) \rightarrow \phi^{\prime}(\mathbf{r})=\phi(\mathbf{r})-\mathrm{i} 4 Q b \ln |\mathbf{r}|$. Using subsequently the Poisson equation $\Delta \ln |\mathbf{r}|=2 \pi \delta(\mathbf{r})$ and integrating by parts with vanishing boundary contributions at $|\mathbf{r}| \rightarrow \infty$, one gets

$$
\exp \left(-\beta \mu_{Q}^{\mathrm{ex}}\right)=\left\langle\mathrm{e}^{\mathrm{i} Q b \phi}\right\rangle
$$

where the average is taken with the usual sine-Gordon action $S(z)$ given by Eq. (2.11). When $Q= \pm 1$, one recovers the previous result (2.13) derived for the plasma constituents. Note the obvious symmetry $\mu_{Q}^{\mathrm{ex}}=\mu_{-Q}^{\mathrm{ex}}$.

An exact formula for the expectation value of the exponential field $\left\langle\mathrm{e}^{\mathrm{i} a \phi}\right\rangle$, where the sine-Gordon parameter $b^{2}$ lies inside the stability region $0 \leq$ $b^{2}<1 / 2$ and $a$ is a free real parameter, was conjectured by Lukyanov and Zamolodchikov in ref. [38. In terms of our notation $a=Q b$ [see Eq. (3.3)], their formula reads

$$
\left\langle\mathrm{e}^{\mathrm{i} Q b \phi}\right\rangle=\left[\frac{\pi z \Gamma\left(1-b^{2}\right)}{\Gamma\left(b^{2}\right)}\right]^{\frac{Q^{2} b^{2}}{1-b^{2}}} \exp \left[I_{b}(Q)\right], \quad|Q|<\frac{1}{2 b^{2}}
$$

with

$$
I_{b}(Q)=\int_{0}^{\infty} \frac{\mathrm{d} t}{t}\left[\frac{\sinh ^{2}\left(2 Q b^{2} t\right)}{2 \sinh \left(b^{2} t\right) \sinh t \cosh \left[\left(1-b^{2}\right) t\right]}-2 Q^{2} b^{2} \mathrm{e}^{-2 t}\right]
$$

The formula was "guessed" on the base of three exactly solvable cases of the sine-Gordon theory: the semi-classical limit $b^{2} \rightarrow 0$, the free-fermion point $b^{2}=1 / 2$ [39] and the special value of $a=b$, see Eq. (2.22). The validity of the formula was supported later by a "reflection" relationship with the imaginary Liouville theory [40], a numerical study of the sine-Gordon model in finite volume [41] and a variational perturbation theory [42. Other checks, provided by the Coulomb-gas representation, are presented in the next two paragraphs.

The integral (3.5) is finite provided that $|Q|<1 /\left(2 b^{2}\right)$; at $|Q|=1 /\left(2 b^{2}\right)$, the integrated function behaves like $1 / t$ for $t \rightarrow \infty$ what causes the logarithmic divergence of the integral. In the Coulomb-gas picture, the interaction Boltzmann factor of the $Q$-charge with an opposite unit plasma charge (counterion) at distance $r, r^{-\beta|Q|}$, is integrable at small $r$ in $2 \mathrm{D}$ if and only if 
$\beta|Q|<2$. The stability region for $\mu_{Q}^{\mathrm{ex}}$ therefore is expected to be $|Q|<2 / \beta$; there is a collapse at $|Q|=2 / \beta$ characterized by $\mu_{Q}^{\mathrm{ex}} \rightarrow-\infty$. With regard to the relationship $\beta=4 b^{2}$ we conclude that the pair of Eqs. (3.4) and (3.5) passes the guest-charge collapse test.

The way in which $\left\langle\mathrm{e}^{\mathrm{i} Q b \phi}\right\rangle$ diverges as $|Q|$ approaches the collapse value $1 /\left(2 b^{2}\right)$ is another check provided by the Coulomb-gas representation. To show this fact, let us attach a hard core of radius $\sigma$ around the guest $Q$ charge. The effect of the $Q$-charge on the species fugacities is the following: $z_{q} \rightarrow z_{q}(\mathbf{r})=z_{q}|\mathbf{r}|^{\beta Q q} \theta(|\mathbf{r}|-\sigma)$, where $\theta$ is the Heaviside step function. The procedure analogous to that outlined between Eqs. (3.1) and (3.3) leads to

$$
\exp \left[-\beta \mu_{Q}^{\mathrm{ex}}(\sigma)\right]=\left\langle\mathrm{e}^{\mathrm{i} Q b \phi(\mathbf{0})}\right\rangle_{\sigma}
$$

where the average is taken with the action

$$
S_{\sigma}(z)=S(z)+z \int_{r<\sigma} \mathrm{d}^{2} r\left[\mathrm{e}^{\mathrm{i} b \phi(\mathbf{r})}+\mathrm{e}^{-\mathrm{i} b \phi(\mathbf{r})}\right]
$$

We expand $\left\langle\mathrm{e}^{\mathrm{i} Q b \phi(\mathbf{0})}\right\rangle_{\sigma}$ in the lowest $\sigma$-order around the sine-Gordon action $S(z)$ :

$$
\left\langle\mathrm{e}^{\mathrm{i} Q b \phi(\mathbf{0})}\right\rangle_{\sigma}=\left\langle\mathrm{e}^{\mathrm{i} Q b \phi}\right\rangle-z \int_{r<\sigma} \mathrm{d}^{2} r\left[\left\langle\mathrm{e}^{\mathrm{i} Q b \phi(\mathbf{0})} \mathrm{e}^{\mathrm{i} b \phi(\mathbf{r})}\right\rangle+\left\langle\mathrm{e}^{\mathrm{i} Q b \phi(\mathbf{0})} \mathrm{e}^{-\mathrm{i} b \phi(\mathbf{r})}\right\rangle\right]+\cdots
$$

Due to the symmetry $\mu_{Q}^{\mathrm{ex}}(\sigma)=\mu_{-Q}^{\mathrm{ex}}(\sigma)$, it is sufficient to consider the case $Q>0$. Applying in Eq. (3.8) the short-distance formula (2.15), the leading $\sigma$-correction is obtained in the form

$$
\left\langle\mathrm{e}^{\mathrm{i} Q b \phi(\mathbf{0})}\right\rangle_{\sigma} \sim\left\langle\mathrm{e}^{\mathrm{i} Q b \phi}\right\rangle-\pi z\left\langle\mathrm{e}^{\mathrm{i}(Q-1) b \phi}\right\rangle \frac{\sigma^{2-4 Q b^{2}}}{1-2 Q b^{2}} \quad \text { as } \sigma \rightarrow 0
$$

Close to the collapse value of the guest charge, i.e. when $Q=1 /\left(2 b^{2}\right)-\epsilon$ with $\epsilon \rightarrow 0^{+}$, one expands

$$
\frac{\sigma^{2-4 Q b^{2}}}{1-2 Q b^{2}}=\frac{\sigma^{4 b^{2} \epsilon}}{2 b^{2} \epsilon}=\frac{1}{2 b^{2} \epsilon}+2 \ln \sigma+O(\epsilon)
$$

Since the regularized $\left\langle\mathrm{e}^{\mathrm{i} Q b \phi(\mathbf{0})}\right\rangle_{\sigma}$ has to be finite at the collapse $Q=1 /\left(2 b^{2}\right)$, it follows from Eqs. (3.9) and (3.10) that the singular behavior

$$
\lim _{\epsilon \rightarrow 0^{+}}\left\langle\exp \left[\mathrm{i}\left(\frac{1}{2 b}-\epsilon b\right) \phi\right]\right\rangle \sim \frac{\pi z}{2 b^{2} \epsilon}\left\langle\exp \left[\mathrm{i}\left(\frac{1}{2 b}-b\right) \phi\right]\right\rangle
$$

must take place. It is shown in the Appendix that the singular behavior (3.11) is indeed reproduced by the conjectured Eqs. (3.4) and (3.5). 


\section{Two guest charges in the electrolyte}

Let us put two point-like particles into the bulk of the Coulomb plasma, the one with the charge $Q_{1}$ at the point $\mathbf{r}_{1}$ and the other with the charge $Q_{2}$ at the point $\mathbf{r}_{2}$. The electrostatic potential induced by these two charges at a point $\mathbf{r} \in R^{2}$ is equal to $-Q_{1} \ln \left|\mathbf{r}-\mathbf{r}_{1}\right|-Q_{2} \ln \left|\mathbf{r}-\mathbf{r}_{2}\right|$. The constant species fugacities $z_{ \pm}$are thus modified as follows: $z_{q} \rightarrow z_{q}^{(2)}(\mathbf{r})=z\left|\mathbf{r}-\mathbf{r}_{1}\right|^{\beta Q_{1} q}\left|\mathbf{r}-\mathbf{r}_{2}\right|^{\beta Q_{2} q}$. The excess chemical potential of the guest 1,2-charges as a whole is given by

$$
\exp \left[-\beta \mu_{Q_{1} Q_{2}}^{\mathrm{ex}}\left(\mathbf{r}_{1}, \mathbf{r}_{2}\right)\right]=\left|\mathbf{r}_{1}-\mathbf{r}_{2}\right|^{\beta Q_{1} Q_{2}} \frac{\Xi\left[z_{q}^{(2)}(\mathbf{r})\right]}{\Xi(z)}
$$

Under the Hubbard-Stratonovich transformation, the sine-Gordon representation of $\Xi\left[z_{q}^{(2)}(\mathbf{r})\right]$ takes the standard functional form of Eq. (2.10) with the action

$$
\begin{aligned}
S^{(2)}(z)= & -\int_{\Lambda} \mathrm{d}^{2} r\left(\frac{1}{16 \pi} \phi \Delta \phi+z\left|\mathbf{r}-\mathbf{r}_{1}\right|^{4 Q_{1} b^{2}}\left|\mathbf{r}-\mathbf{r}_{2}\right|^{4 Q_{2} b^{2}} \mathrm{e}^{\mathrm{i} b \phi}\right. \\
& \left.+z\left|\mathbf{r}-\mathbf{r}_{1}\right|^{-4 Q_{1} b^{2}}\left|\mathbf{r}-\mathbf{r}_{2}\right|^{-4 Q_{2} b^{2}} \mathrm{e}^{-\mathrm{i} b \phi}\right)
\end{aligned}
$$

Shifting the scalar field $\phi(\mathbf{r}) \rightarrow \phi^{\prime}(\mathbf{r})=\phi(\mathbf{r})-\mathrm{i} 4 Q_{1} b \ln \left|\mathbf{r}-\mathbf{r}_{1}\right|-\mathrm{i} 4 Q_{2} b \ln \left|\mathbf{r}-\mathbf{r}_{2}\right|$, applying the Poisson equations $\Delta \ln \left|\mathbf{r}-\mathbf{r}_{j}\right|=2 \pi \delta\left(\mathbf{r}-\mathbf{r}_{j}\right)(j=1,2)$ and integrating by parts, one arrives at

$$
\exp \left[-\beta \mu_{Q_{1} Q_{2}}^{\mathrm{ex}}\left(\mid \mathbf{r}_{1}-\mathbf{r}_{2}\right) \mid\right]=\left\langle\mathrm{e}^{\mathrm{i} Q_{1} b \phi\left(\mathbf{r}_{1}\right)} \mathrm{e}^{\mathrm{i} Q_{2} b \phi\left(\mathbf{r}_{2}\right)}\right\rangle
$$

where the average is taken with the usual sine-Gordon action (2.11). The effective interaction energy between the guest 1,2-charges is defined by

$$
E_{Q_{1} Q_{2}}\left(\left|\mathbf{r}_{1}-\mathbf{r}_{2}\right|\right)=\mu_{Q_{1} Q_{2}}^{\mathrm{ex}}\left(\left|\mathbf{r}_{1}-\mathbf{r}_{2}\right|\right)-\mu_{Q_{1}}^{\mathrm{ex}}-\mu_{Q_{2}}^{\mathrm{ex}}
$$

With respect to the relation (3.3), it holds

$$
\exp \left[-\beta E_{Q_{1} Q_{2}}\left(\left|\mathbf{r}_{1}-\mathbf{r}_{2}\right|\right)\right]=\frac{\left\langle\mathrm{e}^{\mathrm{i} Q_{1} b \phi\left(\mathbf{r}_{1}\right)} \mathrm{e}^{\mathrm{i} Q_{2} b \phi\left(\mathbf{r}_{2}\right)}\right\rangle}{\left\langle\mathrm{e}^{\mathrm{i} Q_{1} b \phi}\right\rangle\left\langle\mathrm{e}^{\mathrm{i} Q_{2} b \phi}\right\rangle}
$$

At asymptotically large distance $\left|\mathbf{r}_{1}-\mathbf{r}_{2}\right| \rightarrow \infty$, the two-point correlator $\left\langle\mathrm{e}^{\mathrm{i} Q_{1} b \phi\left(\mathbf{r}_{1}\right)} \mathrm{e}^{\mathrm{i} Q_{2} b \phi\left(\mathbf{r}_{2}\right)}\right\rangle$ decouples onto the product $\left\langle\mathrm{e}^{\mathrm{i} Q_{1} b \phi}\right\rangle\left\langle\mathrm{e}^{\mathrm{i} Q_{2} b \phi}\right\rangle$ and so the interaction energy goes to zero as it should be. From Eq. (4.5) one then gets

$$
-\beta E_{Q_{1} Q_{2}}\left(\left|\mathbf{r}_{1}-\mathbf{r}_{2}\right|\right) \sim \frac{\left\langle\mathrm{e}^{\mathrm{i} Q_{1} b \phi\left(\mathbf{r}_{1}\right)} \mathrm{e}^{\mathrm{i} Q_{2} b \phi\left(\mathbf{r}_{2}\right)}\right\rangle}{\left\langle\mathrm{e}^{\mathrm{i} Q_{1} b \phi}\right\rangle\left\langle\mathrm{e}^{\mathrm{i} Q_{2} b \phi}\right\rangle}-1, \quad\left|\mathbf{r}_{1}-\mathbf{r}_{2}\right| \rightarrow \infty
$$

This means that the asymptotic large-distance behavior of the effective interaction energy between the guest particles is related to the large-distance 
behavior of the corresponding two-point correlation function of exponential fields associated with the 2D sine-Gordon theory.

For the 2D Euclidean sine-Gordon model, like for any integrable 2D theory, the two-point correlation function of local operators $\mathcal{O}_{a}$ ( $a$ is a free parameter) can be formally expressed as an infinite convergent series over multi-particle intermediate states 43 ,

$$
\begin{aligned}
& \left\langle\mathcal{O}_{a}(\mathbf{r}) \mathcal{O}_{a^{\prime}}\left(\mathbf{r}^{\prime}\right)\right\rangle=\sum_{N=0}^{\infty} \frac{1}{N !} \sum_{\epsilon_{1}, \ldots, \epsilon_{N}} \int_{-\infty}^{\infty} \frac{\mathrm{d} \theta_{1} \cdots \mathrm{d} \theta_{N}}{(2 \pi)^{N}} F_{a}\left(\theta_{1}, \ldots, \theta_{N}\right)_{\epsilon_{1} \cdots \epsilon_{N}} \\
& { }^{\epsilon_{N} \cdots \epsilon_{1}} F_{a^{\prime}}\left(\theta_{N}, \ldots, \theta_{1}\right) \exp \left(-\left|\mathbf{r}-\mathbf{r}^{\prime}\right| \sum_{j=1}^{N} m_{\epsilon_{j}} \cosh \theta_{j}\right)
\end{aligned}
$$

where $\epsilon$ indexes the particles [say $\epsilon=+(-)$ for a soliton (antisoliton) and $\epsilon=j$ for a $B_{j}$-breather] and the rapidity $\theta \in(-\infty, \infty)$ parametrizes the energy and the momentum of the corresponding particle. The form factors

$$
\begin{aligned}
F_{a}\left(\theta_{1}, \ldots, \theta_{N}\right)_{\epsilon_{1} \cdots \epsilon_{N}} & =\left\langle 0\left|\mathcal{O}_{a}(\mathbf{0})\right| Z_{\epsilon_{1}}\left(\theta_{1}\right), \ldots, Z_{\epsilon_{N}}\left(\theta_{N}\right)\right\rangle \\
\epsilon_{N} \cdots \epsilon_{1} & F_{a^{\prime}}\left(\theta_{N}, \ldots, \theta_{1}\right)=\left\langle Z_{\epsilon_{N}}\left(\theta_{N}\right), \ldots, Z_{\epsilon_{1}}\left(\theta_{1}\right)\left|\mathcal{O}_{a^{\prime}}(\mathbf{0})\right| 0\right\rangle
\end{aligned}
$$

are the matrix elements of the operator at the origin, between an $N$-particle in-state (being a linear superposition of free one-particle states $\left|Z_{\epsilon}(\theta)\right\rangle$ ) and the vacuum. The first $N=0$ term of the series expansion (4.7) corresponds to the decoupling $\left\langle\mathcal{O}_{a}\right\rangle\left\langle\mathcal{O}_{a^{\prime}}\right\rangle$.

The form-factor representation (4.7) is particularly useful in the limit $\left|\mathbf{r}-\mathbf{r}^{\prime}\right| \rightarrow \infty$ where the dominant contribution to the truncated correlation function $\left\langle\mathcal{O}_{a}(\mathbf{r}) \mathcal{O}_{a^{\prime}}\left(\mathbf{r}^{\prime}\right)\right\rangle-\left\langle\mathcal{O}_{a}\right\rangle\left\langle\mathcal{O}_{a^{\prime}}\right\rangle$ comes from a multi-particle state with the minimum value of the total particle mass $\sum_{j=1}^{N} m_{\epsilon_{j}}$, at the point of vanishing rapidities. As was already mentioned, the lightest particle in the stability region $0 \leq b^{2}<1 / 2$ is the elementary breather $B_{1}$. For this particle, the one-particle form factors $F_{a}(\theta)_{1}$ and ${ }^{1} F_{a^{\prime}}(\theta)=F_{a^{\prime}}(\theta)_{1}$ of the exponential operator $\mathcal{O}_{a}(\mathbf{r})=\exp [\mathrm{i} a \phi(\mathbf{r})]$ were calculated in refs. [44, 45]:

$$
F_{a}(\theta)_{1} \equiv\left\langle 0\left|\mathrm{e}^{\mathrm{i} a \phi}\right| B_{1}(\theta)\right\rangle=-\mathrm{i}\left\langle\mathrm{e}^{\mathrm{i} a \phi}\right\rangle \sqrt{\pi \lambda} \frac{\sin (\pi \xi a / b)}{\sin (\pi \xi)}
$$

where

$$
\lambda=\frac{4}{\pi} \sin (\pi \xi) \cos \left(\frac{\pi \xi}{2}\right) \exp \left\{-\int_{0}^{\pi \xi} \frac{\mathrm{d} t}{\pi} \frac{t}{\sin t}\right\}
$$

and $\xi$ is defined in Eq. (2.16). Since the form factor (4.10) does not depend on the rapidity, the integration over $\theta$ in (4.7) can be done explicitly by using the relation

$$
\int_{-\infty}^{\infty} \frac{\mathrm{d} \theta}{2} \mathrm{e}^{-r m_{1} \cosh \theta}=K_{0}\left(m_{1} r\right) \sim\left(\frac{\pi}{2 m_{1} r}\right)^{1 / 2} \exp \left(-m_{1} r\right) \quad \text { as } r \rightarrow \infty
$$


where $K_{0}$ is the modified Bessel function of second kind [46]. On the base of the asymptotic equivalence (4.6), the large-distance behavior of the effective interaction energy is finally found in the form

$$
\beta E_{Q_{1} Q_{2}}(r) \sim\left[Q_{1}\right]\left[Q_{2}\right] \lambda\left(\frac{\pi}{2 m_{1} r}\right)^{1 / 2} \exp \left(-m_{1} r\right), \quad r \rightarrow \infty
$$

Here, the symbol $[Q]$ stands for the ratio

$$
[Q]=\frac{\sin (\pi \beta Q /(4-\beta))}{\sin (\pi \beta /(4-\beta))}
$$

Using the thermodynamic formulas derived at the end of Section 2, the mass $m_{1}$ (which plays the role of the inverse charge-charge correlation length of the Coulomb-gas particles) is expressible as

$$
m_{1}=\kappa\left[\frac{\sin (\pi \beta /(4-\beta))}{\pi \beta /(4-\beta)}\right]^{1 / 2}
$$

where $\kappa=\sqrt{2 \pi \beta n}$ denotes the inverse Debye length. The $\beta$-dependence of the parameter $\lambda$, defined by Eq. (4.11), reads

$$
\lambda=\frac{4}{\pi} \sin \left(\frac{\pi \beta}{4-\beta}\right) \cos \left(\frac{\pi \beta}{2(4-\beta)}\right) \exp \left\{-\int_{0}^{\frac{\pi \beta}{4-\beta}} \frac{\mathrm{d} t}{\pi} \frac{t}{\sin t}\right\}
$$

An interesting feature of the result (4.13) is that the effective interaction energy factorizes into the product of separate charge contributions $[Q]$ from each of the guest particles.

We would like to emphasize that the asymptotic formula (4.13) was derived for the guest particles of point-like nature. Its rigorous validity is therefore restricted to such guest charges which do not collapse with an opposite unit counterion from the electrolyte, i.e. to the values $\left|Q_{1}\right|,\left|Q_{2}\right|<2 / \beta$. Since the function $[Q]$ is analytic at every real $Q$, the stability border $|Q|=2 / \beta$ does not represent an exceptional point at which a singularity emerges [like it was in the case of the excess chemical potential determined by Eqs. (3.3)(3.5)]. The explanation of this important fact follows from the definition (4.4) of the effective interaction energy: if one of the guest charges passes or is beyond its collapse value, say $\left|Q_{1}\right| \geq 2 / \beta$, both excess chemical potentials $\mu_{Q_{1} Q_{2}}^{\mathrm{ex}}\left(\left|\mathbf{r}_{1}-\mathbf{r}_{2}\right|\right)$ and $\mu_{Q_{1}}^{\mathrm{ex}}$ tend to $-\infty$ in such a way that their difference is expected to keep a finite value. We therefore suggest that the formula (4.13) remains valid for arbitrary real values of $Q_{1}$ and $Q_{2}$, and corresponds to the limit of a small hard-core radius $\sigma$ (such that $m_{1} \sigma<<1$ ) around the guest charges. In what follows, we shall refer to this conjecture as "the regularization hypothesis". 
The interaction energy $E_{Q_{1} Q_{2}}(r)$ is repulsive (attractive) at asymptotically large distance $r$ when it goes to zero from above (below). When the amplitudes of the guest charges are the same, i.e. $\left|Q_{1}\right|=\left|Q_{2}\right|$, the interaction energy (4.13) exhibits the vacuum-type behavior: it is repulsive for $Q_{1}=Q_{2}$ and, since $[-Q]=-[Q]$, attractive for $Q_{1}=-Q_{2}$. The situation is more complex when the amplitudes of the guest charges differ from one another. Let us analyze the plot of $[Q]$ as the function of (say positive) bare charge $Q$ for a fixed value of $\beta$ from the stability range $\langle 0,2)$. In the interval $Q \in\langle 0,(2 / \beta)-(1 / 2)\rangle,[Q]$ increases monotonically from 0 to its maximum at the end-point of this interval. In the subsequent interval $Q \in\langle(2 / \beta)-(1 / 2),(4 / \beta)-1\rangle$, which contains the stability border $Q=2 / \beta$, $[Q]$ is the decreasing function of $Q$ but still keeps the positive sign of $Q$. Such behavior means physically that by increasing the bare charge of one of the guest particles the effective interaction energy weakens which is a counterintuitive phenomenon. The function $[Q]$ changes the sign when $Q$ passes the value $(4 / \beta)-1$; under the assumption of validity of the regularization hypothesis, this indicates an effective change of the sign of the bare charge $Q$. Appropriate combinations of the $Q_{1}, Q_{2}$-charges in the factorized relation (4.13) can therefore lead to an effective attraction (repulsion) between likecharged (oppositely-charged) guest particles. The described scenario repeats itself when increasing $Q$ due to the periodicity relation $[Q]=[Q+(8 / \beta)-2]$.

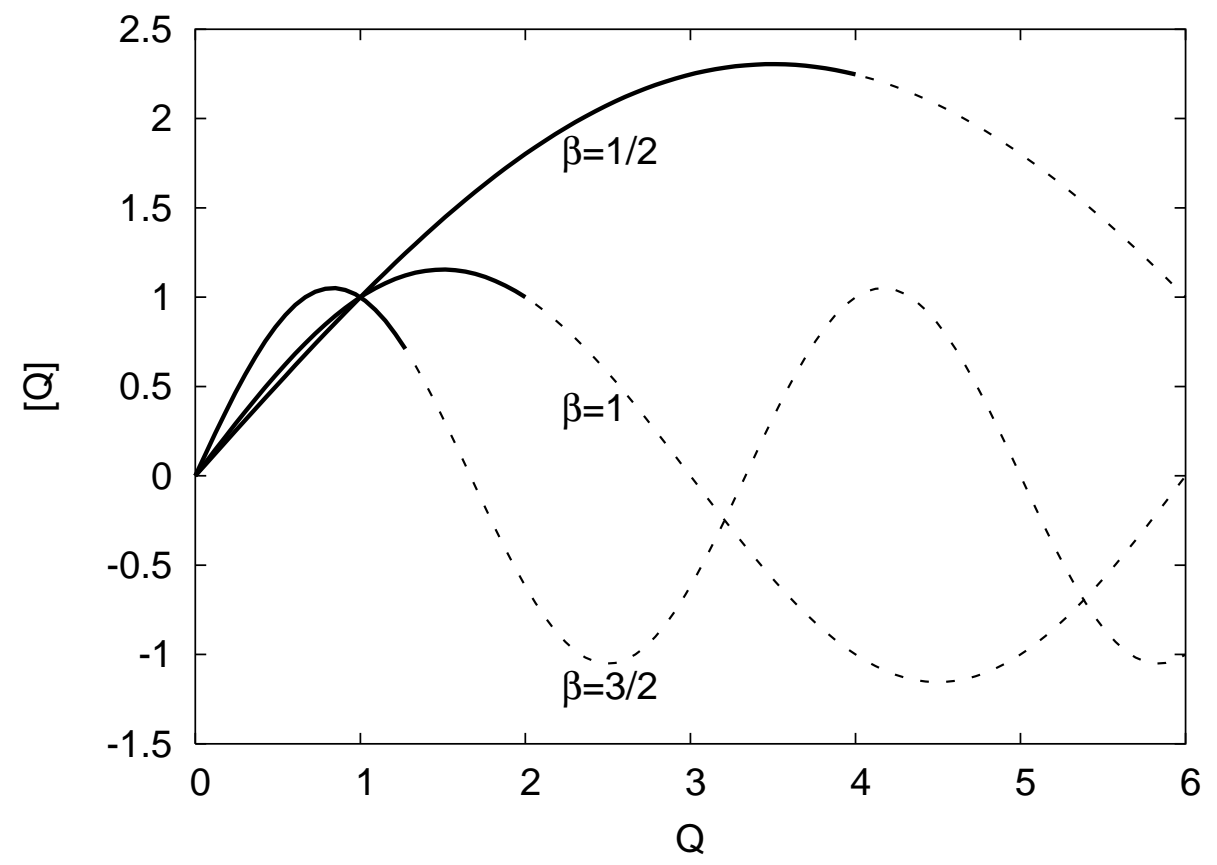

Fig. 1. The function $[Q]$ versus the bare charge $Q$ for various values of $\beta$. 
The above discussed plot of $[Q]$ versus the (positive) bare charge $Q$ is presented graphically for various values of the electrolyte coupling constant $\beta$ in Fig. 1. The solid-line parts of the plots correspond to the interval $0<Q<2 / \beta$ for which the formula (4.13) is valid rigorously, the dashedline parts of the plots correspond to $Q \geq 2 / \beta$ when the formula (4.13) is applicable under the assumption of the regularization hypothesis.

\section{Renormalized charge}

Let us put one point-like particle of charge $Q$ at the origin $\mathbf{0}$ and look for the evoked density profiles $n_{q}(\mathbf{r})$ of the electrolyte species $q= \pm 1$. One can formally follow the procedure for two guest particles (one of which has the charge $q= \pm 1$ of the electrolyte species) outlined at the beginning of Section 4 , to obtain

$$
n_{q}(\mathbf{r})=z \frac{\left\langle\mathrm{e}^{\mathrm{i} Q b \phi(\mathbf{0})} \mathrm{e}^{\mathrm{i} q b \phi(\mathbf{r})}\right\rangle}{\left\langle\mathrm{e}^{\mathrm{i} Q b \phi}\right\rangle}, \quad q= \pm 1
$$

Note that in the special case $Q=0$ the spatially-homogeneous relation (2.23) is reproduced. At asymptotically large distance $r$ from the guest $Q$ charge, the straightforward application of the form-factor method explained in Section 4 provides the result

$$
n_{q}(r) \sim n_{q}\left\{1-[q][Q] \lambda\left(\frac{\pi}{2 m_{1} r}\right)^{1 / 2} \exp \left(-m_{1} r\right)\right\}, \quad r \rightarrow \infty
$$

where the symbols $[q],[Q]$ are defined by Eq. (4.14) and the parameters $m_{1}$, $\lambda$ by the respective relations (4.15) and (4.16).

The charge density $\rho$ of electrolyte unit charges is defined as $\rho(\mathbf{r})=$ $n_{+}(\mathbf{r})-n_{-}(\mathbf{r})$. Since $[q]=q$ for $q= \pm 1$, one finds that

$$
\rho(r) \sim-n[Q] \lambda\left(\frac{\pi}{2 m_{1} r}\right)^{1 / 2} \exp \left(-m_{1} r\right), \quad r \rightarrow \infty
$$

The average electrostatic potential $\psi$ induced by the guest $Q$-charge is related to the charge-density profile through the 2D Poisson equation,

$$
\Delta \psi(\mathbf{r})=-2 \pi \rho(\mathbf{r})
$$

Inserting here the asymptotic formula (5.3) and considering the circularly symmetric Laplacian $\Delta=r^{-1} \mathrm{~d}_{r}\left(r \mathrm{~d}_{r}\right)$, the dimensionless electric potential $\beta \psi$ can be shown to behave at large distance $r$ from the $Q$-charge as follows

$$
\beta \psi(r) \sim\left(\frac{\kappa}{m_{1}}\right)^{2}[Q] \lambda\left(\frac{\pi}{2 m_{1} r}\right)^{1 / 2} \exp \left(-m_{1} r\right), \quad r \rightarrow \infty
$$


In the Debye-Hückel limit $\beta \rightarrow 0$ and for finite $Q$, it holds $[Q] \sim Q, m_{1} \sim \kappa$ and $\lambda \sim \beta$. Eq. (5.5) thus reduces to the well-known result (see e.g. refs. [1, 28, )

$$
\beta \psi_{\mathrm{DH}}(r) \sim \beta Q\left(\frac{\pi}{2 \kappa r}\right)^{1 / 2} \exp (-\kappa r), \quad r \rightarrow \infty
$$

The asymptotic behaviors (5.5) and (5.6), considered respectively in terms of the dimensionless combinations $m_{1} r$ and $\kappa r$, exhibit the same type of the falloff. This fact confirms the adequacy of the concept of renormalized charge [18] - 25] in the stability weak-coupling regime of the Coulomb gas. Eq. (5.5]) is consistent with Eq. (5.6) provided that one introduces the renormalized charge $Q_{\text {ren }}$ as follows

$$
\beta Q_{\text {ren }}=\left(\frac{\kappa}{m_{1}}\right)^{2}[Q] \lambda
$$

This formula can be simplified to

$$
Q_{\mathrm{ren}}(\beta, Q)=A(\beta) \sin \left(\frac{\pi \beta Q}{4-\beta}\right)
$$

where the positive amplitude $A(\beta)$ is given by

$$
\frac{1}{A(\beta)}=\frac{1}{2}(4-\beta) \sin \left(\frac{\pi \beta}{2(4-\beta)}\right) \exp \left\{\int_{0}^{\frac{\pi \beta}{4-\beta}} \frac{\mathrm{d} t}{\pi} \frac{t}{\sin t}\right\}
$$

As was mentioned above, because of the point-like nature of the guest $Q$-charge the rigorous validity of the result (5.8) is restricted to $|Q|<2 / \beta$. Changing $Q$ from 0 towards positive real values, the renormalized charge (5.8) increases monotonically up to its maximum $A(\beta)$ at the point $Q=$ $(2 / \beta)-(1 / 2)$. Increasing then $Q$ from $(2 / \beta)-(1 / 2)$ up to the stability border $2 / \beta, Q_{\text {ren }}$ paradoxically decreases while still keeping the positive sign of the bare charge $Q$. This scenario resembles the one observed in the Groot's Monte-Carlo simulations of the salt-free (only counterions are present) colloidal cell model 24].

Under the assumption of validity of the regularization hypothesis, one can further increase the value of $Q$ beyond $2 / \beta$ in the relation (5.8). $Q_{\text {ren }}$ changes its positive sign to the negative one at $Q=(4 / \beta)-1$. This change of the sign is closely related to the effect of charge inversion [13]-[17]: since the total screening cloud of electrolyte particles must compensate exactly the bare charge $Q$ of the guest particle (the electroneutrality sum rule), the fact that the charge density (5.3) goes to 0 at asymptotically large distance $r$ from above is the evidence of the charge inversion starting at some distance from the guest $Q$-charge.

The renormalized charge $Q_{\text {ren }}$ is a periodic function of $Q$. This is why going with $Q \rightarrow \infty$ does not imply the saturation of $Q_{\text {ren }}$ at some finite 
value. Instead, $Q_{\text {ren }}$ oscillates between the two finite $\pm A(\beta)$ extremes. With regard to Eq. (5.5), the induced electrostatic potential exhibits the same type of the oscillatory behavior as $Q \rightarrow \infty$ what contradicts the idea of the monotonic electric potential saturation [25].

\section{Conclusion}

Let us summarize briefly the crucial results of the present work.

Section 3 deals with the case of one point-like particle of charge $Q$ (with $|Q|<2 / \beta)$ immersed in the bulk of the stable 2D Coulomb gas. Passing to the sine-Gordon representation, we were able to relate in Eq. (3.3) the excess chemical potential $\mu_{Q}^{\mathrm{ex}}$ of the guest charge to the expectation value of the exponential field. The explicit form of the latter quantity was conjectured by Lukyanov and Zamolodchikov 38, see Eqs. (3.4) and (3.5), and subsequently verified by various methods. Our Coulomb-gas formulation provides two other checks of this conjecture: the guest-charge collapse test at $|Q|=2 / \beta$ and the predicted singular behavior (3.11) of the exponential-field expectation close to the collapse value of $Q=(2 / \beta)-\epsilon\left(\epsilon \rightarrow 0^{+}\right)$.

The problem of two guest point-like particles, charged by $Q_{1}$ and $Q_{2}$ and being at distance $r$ from one another, is studied in Section 4. Using the form-factor method for the two-point correlation functions in the sineGordon formulation of the problem, we have derived the explicit formula (4.13) for the effective interaction energy of the two guest charges $E_{Q_{1} Q_{2}}(r)$ at asymptotically large distance $r \rightarrow \infty$. This formula is valid rigorously for $\left|Q_{1}\right|,\left|Q_{2}\right|<2 / \beta$; in a subspace of this region of charge values we have noticed an anomalous weakening of the effective interaction when one of the guest charges is increasing. Since in the definition (4.4) of $E_{Q_{1} Q_{2}}(r)$ the divergences of chemical potentials (caused by the collapse of guest charge with electrolyte counterions) cancel with each other, we have suggested an extended validity of the formula (4.13) for arbitrary values of $Q_{1}$ and $Q_{2}$ (the regularization hypothesis). Then, under certain circumstances (especially, the inequality $\left|Q_{1}\right| \neq\left|Q_{2}\right|$ must hold and one of the guest charges has to be large enough), the result (4.13) implies an effective attraction (repulsion) between like-charged (oppositely-charged) guest particles.

The adequacy of the concept of renormalized charge was confirmed in Section 5. This is not a surprise: in the whole stability interval of inverse temperatures $0 \leq \beta<2$, the large-distance behavior of two-point correlators is determined by the form-factor of the same particle from the sine-Gordon spectrum, namely the $B_{1}$-breather with the lightest mass $m_{1}$ playing the role of the inverse correlation length of electrolyte species. The large-distance behavior of the induced electric potential (5.5), considered in terms of the dimensionless combination $m_{1} r$, is therefore basically the same in the Debye- 
Hückel limit $\beta \rightarrow 0$ as well as at every point $\beta$ which belongs to the stability interval, up to the renormalized-charge prefactor. The renormalized charge $Q_{\text {ren }}$, considered as a function of the (positive) bare charge $Q$, exhibits a maximum at $Q=(2 / \beta)-(1 / 2)$ which is in the range $|Q|<2 / \beta$ of the rigorous validity of the formula (5.8). Under the assumption of validity of the regularization hypothesis, increasing $Q$ can produce the effect of charge inversion. Going with $Q \rightarrow \infty$ in Eq. (5.8) does not imply the saturation of $Q_{\text {ren }}$ at some finite value. Instead, $Q_{\text {ren }}$ oscillates between two finite extremes. The same property holds for the electric potential in the electrolyte region which contradicts the idea of the monotonic potential saturation [25].

The previous results obtained at the free-fermion (collapse) point $\beta=2$ 28 are different from the present ones concerning the stability interval $0 \leq \beta<2$ in the following aspects. Firstly, the concept of renormalized

charge fails at $\beta=2$. Secondly, at $\beta=2$, when the bare charge $Q \rightarrow \infty$ the induced electrostatic potential saturates monotonically at a finite value in each point of the electrolyte region. The reason for the fundamental differences is obvious. The lightest $B_{1}$-breather disappears from the particle spectrum of the sine-Gordon model just at $\beta=2$, and the asymptotic behavior of two-point correlation functions at this coupling constant is governed by the soliton-antisoliton pair. Since $m_{1} \rightarrow 2 M$ as $\beta \rightarrow 2$, the particle mass in the exponential decay is a continuous function of $\beta$ at $\beta=2$. On the other side, the inverse-power-law asymptotic behavior, which is determined by the formfactor of the dominant particle(s) in the sine-Gordon spectrum, undertakes an abrupt modification when passing through the $\beta=2$ point. The basic qualitative features of the results obtained at the free-fermion point $\beta=2$ are expected to be present also for such $\beta>2$ where the soliton-antisoliton pair exists. It is known [34] that the soliton-antisoliton pair disappears from the sine-Gordon particle spectrum (and the sine-Gordon theory ceases to be massive) at the point $b^{2}=1(\beta=4)$ which corresponds to the KosterlitzThouless transition of infinite order from the conducting (fluid) phase to the insulating phase. We conclude that the $2 \mathrm{D}$ results obtained in the weakcoupling regime of the Coulomb gas $0 \leq \beta<2$ differ substantially from those in the strong-coupling regime $2 \leq \beta<4$.

\section{Appendix}

According to the conjectured Eqs. (3.4) and (3.5), it holds

$$
\lim _{\epsilon \rightarrow 0^{+}}\left\langle\exp \left[\mathrm{i}\left(\frac{1}{2 b}-\epsilon b\right) \phi\right]\right\rangle \sim\left[\frac{\pi z \Gamma\left(1-b^{2}\right)}{\Gamma\left(b^{2}\right)}\right]^{\frac{1}{4 b^{2}\left(1-b^{2}\right)}} \exp \left(I_{1}+I_{2}+I_{3}\right)
$$


where

$$
\begin{aligned}
& I_{1}=\int_{0}^{1} \frac{\mathrm{d} t}{t}\left\{\frac{\sinh (t)}{2 \sinh \left(b^{2} t\right) \cosh \left[\left(1-b^{2}\right) t\right]}-\frac{1}{2 b^{2}} \mathrm{e}^{-2 t}\right\} \\
& I_{2}=\int_{1}^{\infty} \frac{\mathrm{d} t}{t}\left\{\frac{\sinh (t)}{2 \sinh \left(b^{2} t\right) \cosh \left[\left(1-b^{2}\right) t\right]}-1-\frac{1}{2 b^{2}} \mathrm{e}^{-2 t}\right\} \\
& I_{3}=\int_{1}^{\infty} \frac{\mathrm{d} t}{t} \exp \left(-4 b^{2} \epsilon t\right)=-C-\ln \left(4 b^{2} \epsilon\right)+O(\epsilon)
\end{aligned}
$$

and $C$ is the Euler's constant. Using the same Eqs. (3.4) and (3.5) for expressing the expectation value of the exponential field on the rhs of Eq. (3.11), one gets

$$
\lim _{\epsilon \rightarrow 0^{+}} \frac{\left\langle\exp \left[\mathrm{i}\left(\frac{1}{2 b}-\epsilon b\right) \phi\right]\right\rangle}{\left\langle\exp \left[\mathrm{i}\left(\frac{1}{2 b}-b\right) \phi\right]\right\rangle} \sim \frac{\pi z}{2 b^{2} \epsilon} \frac{\Gamma\left(1-b^{2}\right)}{\Gamma\left(b^{2}\right)} \exp \left(I_{1}^{\prime}+I_{2}^{\prime}-C-\ln 2\right)
$$

where

$$
\begin{aligned}
& I_{1}^{\prime}=\int_{0}^{1} \frac{\mathrm{d} t}{t}\left\{\frac{\sinh \left[\left(1-2 b^{2}\right) t\right]}{\sinh (t)}+1-2\left(1-b^{2}\right) \mathrm{e}^{-2 t}\right\} \\
& I_{2}^{\prime}=\int_{1}^{\infty} \frac{\mathrm{d} t}{t}\left\{\frac{\sinh \left[\left(1-2 b^{2}\right) t\right]}{\sinh (t)}-2\left(1-b^{2}\right) \mathrm{e}^{-2 t}\right\}
\end{aligned}
$$

With the aid of the integral representations [46]

$$
\begin{aligned}
C & =\int_{0}^{1} \frac{\mathrm{d} t}{t}\left(1-\mathrm{e}^{-t}\right)-\int_{1}^{\infty} \frac{\mathrm{d} t}{t} \mathrm{e}^{-t} \\
\ln 2 & =\int_{0}^{\infty} \frac{\mathrm{d} t}{t}\left(\mathrm{e}^{-t}-\mathrm{e}^{-2 t}\right)
\end{aligned}
$$

the argument of the exponential on the rhs of Eq. (A.5) can be written as

$$
I_{1}^{\prime}+I_{2}^{\prime}-C-\ln 2=\int_{0}^{\infty} \frac{\mathrm{d} t}{t}\left\{\frac{\sinh \left[\left(1-2 b^{2}\right) t\right]}{\sinh (t)}+\left(2 b^{2}-1\right) \mathrm{e}^{-2 t}\right\}
$$

Finally, considering in Eq. A.5 the integral representation of the logarithm of the Gamma function 46

$$
\ln \Gamma(x)=\int_{0}^{\infty} \frac{\mathrm{d} t}{t} \mathrm{e}^{-t}\left[(x-1)+\frac{\mathrm{e}^{-(x-1) t}-1}{1-\mathrm{e}^{-t}}\right], \quad \operatorname{Re}(x)>0
$$

the proof of the desired formula (3.11) becomes accomplished.

\section{Acknowledgments}

I thank Bernard Jancovici for careful reading of the manuscript and useful comments. The support by Grant VEGA 2/3107/2003 is acknowledged. 


\section{References}

[1] Y. Levin, Rep. Prog. Phys. 65:1577 (2002).

[2] B. V. Derjaguin and L. Landau, Acta Physicochim. URSS 14:633 (1941).

[3] E. J. W. Verwey and J. Th. G. Overbeek, Theory of the Stability of Lyophobic Colloids (Elsevier, New York, 1948).

[4] J. C. Crocker and D. G. Grier, Phys. Rev. Lett. 73:352 (1994).

[5] J. Chakrabarti and H. Löwen, Phys. Rev. E 58:3400 (1998).

[6] G. M. Kepler and S. Fraden, Phys. Rev. Lett. 73:356 (1994).

[7] J. C. Crocker and D. G. Grier, Phys. Rev. Lett. 77:1897 (1996).

[8] N. Grønbech-Jensen, K. M. Beardmore, and P. Pincus, Physica A 261:74 (1998).

[9] E. Allahyarov, I. D'Amico, and H. Löwen, Phys. Rev. E 60:3199 (1999).

[10] N. Ma, S. M. Girvin, and R. Rajaraman, Phys. Rev. E 63:021402 (2001).

[11] T. M. Squires and M. P. Brenner, Phys. Rev. Lett. 85:4976 (2000).

[12] T. M. Squires, J. Fluid Mech. 443:403 (2001).

[13] B. I. Shklovskii, Phys. Rev. E 60:5802 (1999).

[14] H. W. Walker and S. B. Grant, Colloids and Surfaces A 119:229 (1996).

[15] R. Messina, C. Holm, and K. Kremer, Phys. Rev. E 64:021405 (2001).

[16] T. T. Nguyen, A. Y. Grosberg, and B. I. Shklovskii, J. Chem. Phys. 113:1110 (2000).

[17] R. Messina, C. Holm, and K. Kremer, Phys. Rev. Lett. 85:872 (2000).

[18] G. S. Manning, J. Chem. Phys. 51:924 (1969).

[19] S. Alexander, P. M. Chaikin, P. Grant, G. J. Morales, and P. Pincus, J. Chem. Phys. 80:5776 (1984).

[20] H. Löwen, J. Chem. Phys. 100:6738 (1994).

[21] A. Diehl, M. C. Barbosa, and Y. Levin, Europhys. Lett. 53:86 (2001).

[22] E. Trizac, L. Bocquet, and M. Auboy, Phys. Rev. Lett. 89:248301 (2002). 
[23] M. Auboy, E. Trizac, and L. Bocquet, J. Phys. A: Math. Gen. 36:5835 (2003).

[24] R. D. Groot, J. Chem. Phys. 95:9191 (1991).

[25] G. Téllez and E. Trizac, Phys. Rev. E 68:061401 (2003).

[26] F. Cornu and B. Jancovici, J. Stat. Phys. 49:33 (1987).

[27] F. Cornu and B. Jancovici, J. Chem. Phys. 90:2444 (1989).

[28] L. Šamaj, Saturation of Electrostatic Potential: Exactly Solvable 2D Coulomb Models, cond-mat/0501442, to appear in J. Stat. Phys.

[29] L. Šamaj, J. Phys. A: Math. Gen. 36:5913 (2003).

[30] Ph. A. Martin, Rev. Mod. Phys. 60:1075 (1988).

[31] B. Jancovici, J. Stat. Phys. 17:357 (1977).

[32] J. P. Hansen and P. Viot, J. Stat. Phys. 38:823 (1985).

[33] P. Minnhagen, Rev. Mod. Phys. 59:1001 (1987).

[34] A. Zamolodchikov and Al. Zamolodchikov, Ann. Phys. (N.Y.) 120:253 (1979).

[35] C. Destri and H. de Vega, Nucl. Phys. B 358:251 (1991).

[36] Al. Zamolodchikov, Int. J. Mod. Phys. A 10:1125 (1995).

[37] L. Šamaj and I. Travěnec, J. Stat. Phys. 101:713 (2000).

[38] S. Lukyanov and A. Zamolodchikov, Nucl. Phys. B 493:571 (1997).

[39] D. Bernard and A. LeClair, Nucl. Phys. B 426:534 (1994); erratum Nucl. Phys. B 498:619 (1997).

[40] V. Fateev, S. Lukyanov, A. Zamolodchikov and Al. Zamolodchikov, Nucl. Phys. B 516:652 (1998).

[41] Z. Bajnok, L. Palla, G. Takács and F. Wágner, Nucl. Phys. B 587:585 (2000).

[42] W. F. Lu, Phys. Lett. B 602:261 (2004).

[43] F. A. Smirnov, Form-Factors in Completely Integrable Models of Quantum Field Theory (World Scientific, Singapore, 1992). 
[44] S. Lukyanov, Mod. Phys. Lett. A 12:2543 (1997).

[45] S. Lukyanov, Phys. Lett. B 408:192 (1997).

[46] I. S. Gradshteyn and I. M. Ryzhik, Table of Integrals, Series and Products, 5th ed. (Academic Press, London, 1994). 